\title{
VERIFICATION OF A SIMPLE MICROMETEOROLOGICAL METHOD FOR ESTIMATING THE RATE OF GASEOUS MASS TRANSFER FROM THE GROUND TO THE ATMOSPHERE
}

\author{
J.D. WILSON ${ }^{1}$ *, V.R. CATCHPOOLE ${ }^{2}$, O.T. DENMEAD ${ }^{3}$ and G.W. THURTELL ${ }^{4}$ \\ ${ }^{1}$ New Zealand Meteorological Service, P.O. Box 722, Wellington, (New Zealand) \\ ${ }^{2}$ C.S.I.R.O. Division of Tropical Crops and Pastures, The Cunningham Laboratory, \\ St. Lucia, Queensland, 4067 (Australia) \\ ${ }^{3}$ C.S.I.R.O. Division of Environmental Mechanics, P.O. Box 821, Canberra City, A.C.T. \\ (Australia) \\ ${ }^{7}$ Department of Land Resource Science, University of Guelph, Guelph, Ontario (Canada)
}

(Received September 27, 1982; revision accepted March 17, 1983)

\section{ABSTRACT}

Wilson, J.D., Catchpole, V.R., Denmead, O.T. and Thurtell, G.W., 1983. Verification of a simple micrometeorological method for estimating the rate of gaseous mass transfer from the ground to the atmosphere. Agric. Meteorol., 29: 183-189.

Wilson et al proposed a simple micrometeorological method of estimating the rate of gaseous mass transfer to the atmosphere from a small circular plot which required measurements of time-average species-concentration and horizontal windspeed at a single height. This method has been applied here to estimate the rate of volatilization of ammonia from a $25 \mathrm{~m}$ radius plot treated with urea fertilizer. The emission rates thus obtained agreed satisfactorily with estimates based on a mass balance which employed concentration and windspeed measurements at five levels.

\section{INTRODUCTION}

This paper compares two micrometeorological methods of estimating the rate of gaseous mass transfer from the ground to the atmosphere. The methods are applicable to many problems, but will here be compared specifically for the case of ammonia volatilization from urea fertilizer applied to pasture. Both utilize a small (radius $\leqslant 50 \mathrm{~m}$ ) circular plot. Time-average species-concentration and windspeed measurements at the centre of the plot suffice to determine the rate of emission from the plot. The use of a small circular plot is in several ways more practical than the treatment of a large field (upstream extent $\sim 300 \mathrm{~m}$ ) to obtain a shallow constantvertical-flux layer, within which profile or eddy-correlation measurements can determine the rate of emission.

The two circular-plot methods are here labelled the 'integrated horizontal flux' (IHF) method and the 'theoretical profile shape' (TPS) method. The latter is a simplification of the former which reduces the required experimental input by incorporating the prediction of a well-verified surfacelayer dispersion model as to the shape of the concentration profile at the centre of the plot.

\footnotetext{
* Present address: Department of Land Resource Science, University of Guelph, Guelph, Ontario, Canada.
} 
Both methods and the dispersion model used to develop the TPS method are reviewed. The IHF and TPS methods were applied to estimate the rate of volatilization of ammonia from a $25 \mathrm{~m}$ radius plot which was fertilised with urea. The experimental data is used here for the purpose of comparing the IHF and TPS methods, and we are not concerned with interpretation of the estimated time series of ammonia volatilization rate.

\section{THE INTEGRATED HORIZONTAL FLUX METHOD}

Let the circular plot have radius $R$. Assume that, seen from above, trajectories from the edge across the plot exhibit little lateral meandering. In that case, the situation may be considered to be two dimensional, and at the centre of the plot the observer is, in effect, a distance $R$ downwind from the leading edge of a continuous ground-level source of infinite crosswind extent. All material emitted upstream must eventually pass by the observer. This time-average mass balance may be expressed

$\int_{0}^{R} Q(x) \mathrm{d} x=\int_{0}^{\infty} \bar{F}_{x}(z) \mathrm{d} z$

where $x$ and $z$ are the horizontal and vertical coordinates, respectively, $Q(x)$ is the time-average rate of emission, and $\bar{F}_{x}(z)$ is the time-average horizontal flux density. Neglecting the turbulent horizontal flux we may write $\vec{F}_{x}=\bar{c} \bar{s}$ where $\bar{c}$ and $\bar{s}$ are the time-average concentration and windspeed. Then, if $Q$ is spatially uniform

$Q^{\mathrm{IHF}}=\frac{1}{R} \int_{0}^{\infty} \bar{c} \bar{s} \mathrm{~d} z$

Equation 1 is the basis of the 'integrated horizontal flux' method. To determine $Q$, windspeed and concentration must be measured at a number of points along the vertical at the centre of the plot and the integral evaluated.

\section{THE THEORETICAL PROFILE SHAPE METHOD}

Using a trajectory-simulation (TS) model of turbulent dispersion, Wilson et al. (1982, hereafter referred to as WTKB) investigated the vertical profile of horizontal flux $\bar{F}_{x}(z)$ at the centre of a small circular plot, varying the plot radius $R$, roughness length $z_{o}$, and Monin -Obukhov stability length $L$.

The TS model is based on Taylor's (1921) statistical theory of dispersion which implies that for two-dimensional dispersion in the horizontally homogeneous surface-layer the important velocity statistics are the mean horizontal windspeed $\vec{s}(z)$, the mean vertical velocity $\bar{w}(=0)$, the standard deviation of vertical velocity $\sigma_{w}=\left(\overline{w^{2}}\right)^{1 / 2}$, and the Lagrangian timescale 
$\tau_{L}(z)$ which is a measure of the persistence of the vertical velocity of a marked fluid element. A large number of particle trajectories from the source to the collector are simulated, each by a series of steps $\Delta x, \Delta z$ which are calculated in such a way as to incorporate the correct values of $\vec{s}(z), \sigma_{w}$, and $\tau_{L}(z)$. From these many trajectories, the concentration profile may be obtained. Wilson et al. (1981) showed that with a suitable choice for the profile of $\tau_{L}(z)$ (which is not directly measurable with present techniques) the TS model is in excellent agreement with observations of short range dispersion over a very wide range of stability conditions.

When applied to the problem of turbulent dispersion from a small plot, the TS model was found by WTKB to indicate that for given $z_{o}, R$ there is always a height ZINST at which the normalised horizontal flux $\bar{F}_{x} / Q$ is very insensitive to the only remaining parameter, $L$. Accordingly, WTKB suggested the theoretical profile shape (TPS) method. For $R=20 \mathrm{~m}$ and $R=50 \mathrm{~m}$ they gave ZINST as a function of $z_{o}$ and evaluated the narrow range within which $\bar{F}_{x}(Z I N S T) / Q$ must lie. The TPS method is effectively a simplification of the IHF method so that concentration and windspeed need be measured at a single height alone. The roughness length is determined, height ZINST chosen (depending on $z_{o}, R$ ) and the time average windspeed and concentration at ZINST then determine $Q$ within fairly narrow limits. This will be illustrated in a later section.

\section{THE EXPERIMEN'T}

The aim of the experiment was to assess the magnitude of ammonia loss from urea fertilizer applied to pasture. The experimental field was 1.5 ha in area, nearly level, and supported a dense pasture of a tropical grass, Setaria sphacelata CV. Nandi, which was mowed shortly before the commencement of the experiment. Other pasture surrounded the experimental field giving a wind fetch over similar terrain of $200 \mathrm{~m}$ or more.

The fertilizer was spread over a circle of $25 \mathrm{~m}$ radius. Windspeeds were measured at heights of $0.3,0.5,0.9,1.7$ and $2.5 \mathrm{~m}$ at the centre of the circle using sensitive cup anemometers. Ammonia concentrations at the same points were obtained by drawing air at $12.51 \mathrm{~min}^{-1}$ through traps containing $80 \mathrm{ml}$ of $0.2 \mathrm{~N}$ sulphuric acid. The contents of the traps were subsequently analysed for ammonia by an indo-phenol method.

For the first 4 days of the experiment an almost continuous series of 2 -h time-average wind and concentration profiles was obtained. Measurements were made less frequently over the next 5 days. During the experiment the estimated rate of loss of $\mathrm{NH}_{3}$ ranged from a minimum of $0.3 \mu \mathrm{g} \mathrm{m}^{-2} \mathrm{~s}^{-1}$ to a maximum of $32 \mu \mathrm{g} \mathrm{m}^{-2} \mathrm{~s}^{-1}$ (two orders of magnitude).

\section{COMPARISON OF THE IHF AND TPS METHODS}

In order to apply the TPS method it was necessary to determine the roughness length $z_{o}$ and the displacement height $d$. This was done by forming 
an average wind profile from all (38) runs during which the lowest measured mean windspeed exceeded $1 \mathrm{~m} \mathrm{~s}^{-1}$ and choosing the value of $d(0.15 \mathrm{~m})$ which gave the most closely logarithmic profile. Extrapolation to $\bar{s}=0$ gave $z_{o}=0.005 \mathrm{~m}$.

Since the numbers appropriate to a $25 \mathrm{~m}$ radius plot were not given by WTKB, the TS model was run with $R=25 \mathrm{~m}, z_{o}=0.005 \mathrm{~m}$, and Monin Obukhov lengths of $-5 \mathrm{~m}, \infty,+5 \mathrm{~m}$ (extremely unstable, neutral, and extremely stable stratification). Figure 1 compares the normalised concentration profiles $\bar{c} u_{*} / \mathrm{k} Q$ (where $u_{*}$ is the friction velocity and $\mathrm{k}$ is von Karman's constant) predicted by the TS model with the shape of the average observed concentration profile. The latter was obtained by calculating the ratios $\bar{c}(z) / \bar{c}(0.90 \mathrm{~m})$ for each experiment and averaging these over all experiments. The observed profile is matched to the TS model profiles at $z=0.90 \mathrm{~m}$. Given the reasonable agreement in shape of the theoretical and observed profiles over the height range within which most of the transport of $\mathrm{NH}_{3}$ past the centre of the source is occurring, corresponding agreement can be expected between the TPS and IHF estimates of source strength.

Figure 2 shows the predictions of the TS model for the vertical profile of the normalised horizontal flux at the centre of the plot. Note that at the

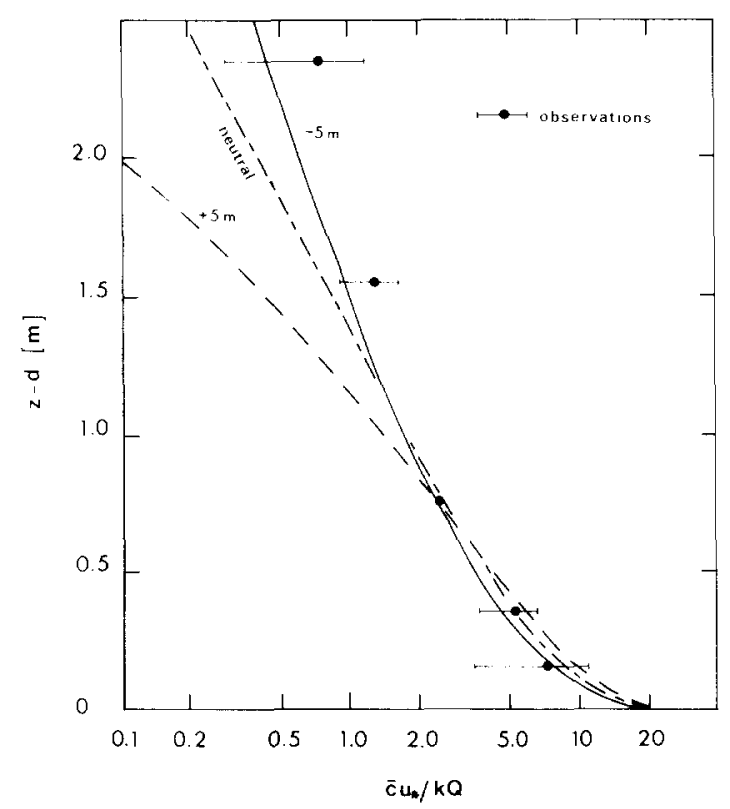

Fig. 1. Comparison of the shape of the observed normalised concentration profile at the centre of a surface disc source of radius $R=25 \mathrm{~m}$ with the profile predicted by the TS model. $u_{*}$ is the friction velocity, $\mathrm{k}$ is von Karman's constant. TS model predictions are for roughness length $z_{o}=0.005 \mathrm{~m}$ and Monin-Obukhov length $L=-5 \mathrm{~m}, \infty$, $+5 \mathrm{~m}$. The observations are matched to the model prediction at $z-\mathrm{d}=0.75 \mathrm{~m}$ and the error bars give \pm 1 sample standard deviation. 


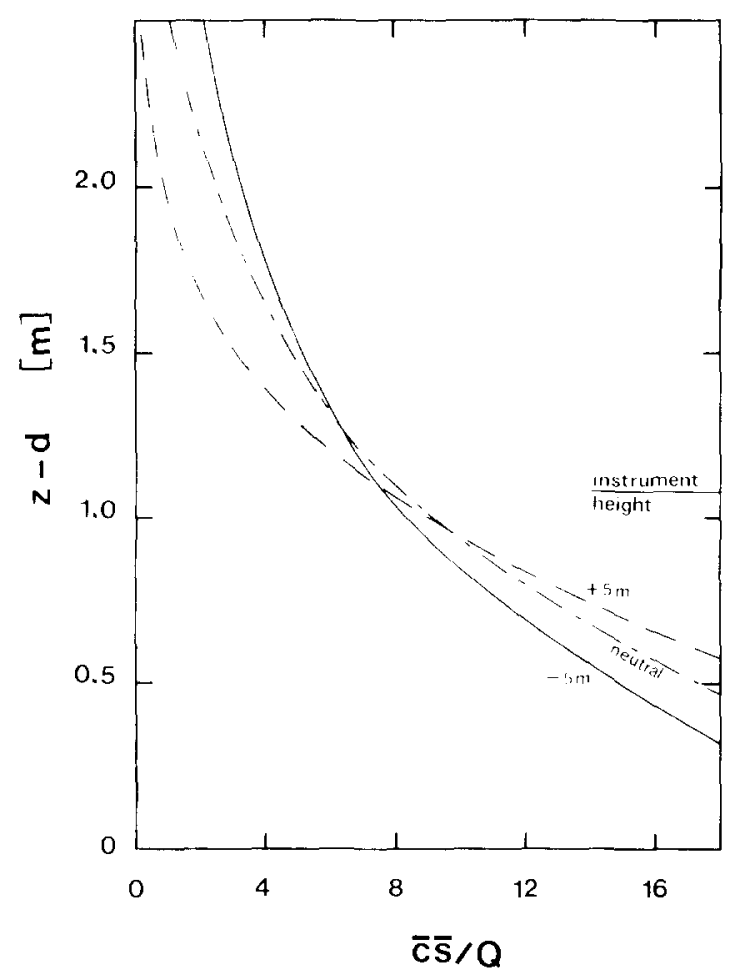

Fig. 2. Predictions of the TS model for the vertical profile of normalised horizontal flux density $\bar{c} s / Q$ at the centre of a surface disc source of radius $R=25 \mathrm{~m}$. Roughness length $z_{0}=0.005 \mathrm{~m}$, Monin-Obukhov length $L=-5 \mathrm{~m}, \infty,+5 \mathrm{~m}$. The profiles for $L=-5 \mathrm{~m}$, $+5 \mathrm{~m}$ intersect at $z-d=1.08 \mathrm{~m}$.

height labelled 'instrument height' the value of $\bar{F}_{x} / Q=\bar{c} \bar{s} / Q$ lies between 7.6 (stable/unstable) and 8.2 (neutral). In this case the procedure of the TPS method is to adopt a measurement height ZINST $-d=1.08 \mathrm{~m}$ (ZINST $=1.23 \mathrm{~m})$ and to assume that measured values of $\bar{F}_{x} / Q$ at this height must lie in the range $7.6 \leqslant \bar{c} \bar{s} / Q^{\mathrm{TPS}} \leqslant 8.2$.

The 'measured' value of $\bar{c} \bar{s}$ at $z=Z I N S T=1.23 \mathrm{~m}$ was obtained by interpolation from the measured profile and divided by $n$, where $7.6 \leqslant n \leqslant 8.2$. An objective (though necessarily very simple) scheme to choose $n$ was employed. If cloud was noted at the time of the experiment $n=8.2$. On clear days $n=8.2$ in early morning or late afternoon; at other times $n=$ 7.9 unless $\bar{s}(2.5)<2.0 \mathrm{~m} \mathrm{~s}^{-1}$ in which case $n=7.6$. On clear nights if $\bar{s}(2.5)<2.0 \mathrm{~m} \mathrm{~s}^{-1} n=7.6$, otherwise $n=8.2$. Of the 62 runs available, 7 were rejected because there was difficulty in interpolating a value for $\bar{c} \bar{s}$ at $1.23 \mathrm{~m}$ owing to the presence of one or more suspect measurements.

The IHF values of the source strength $Q^{\mathrm{IIF}}$ are obtained from eq. 1 . However, the measurements provide no information about the contribution to the integral from below $z=0.3 \mathrm{~m}$ or above $z=2.5 \mathrm{~m}$. Although the 


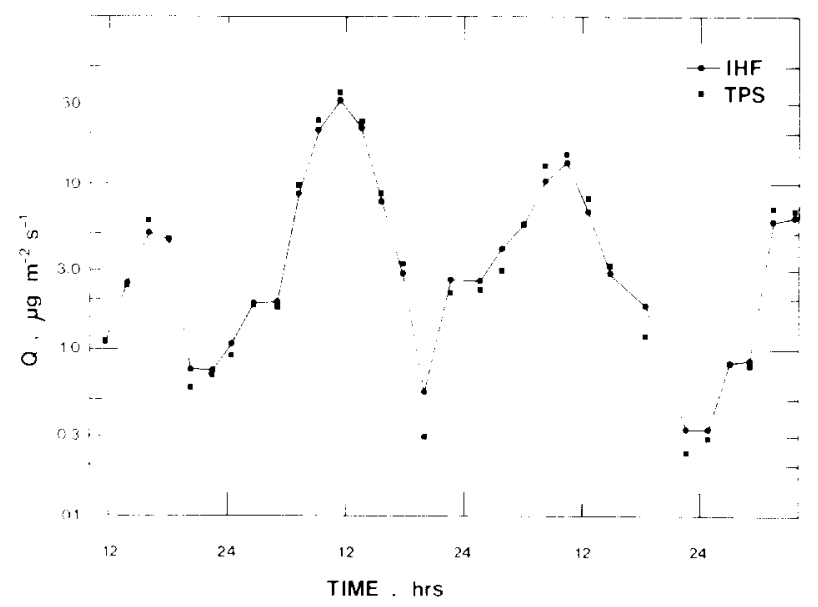

Fig. 3. The rate of loss of ammonia from a circular plot of fertilized grass of radius $25 \mathrm{~m}$ as a function of time. • estimated using the IHF method; - estimated using the TPS method.

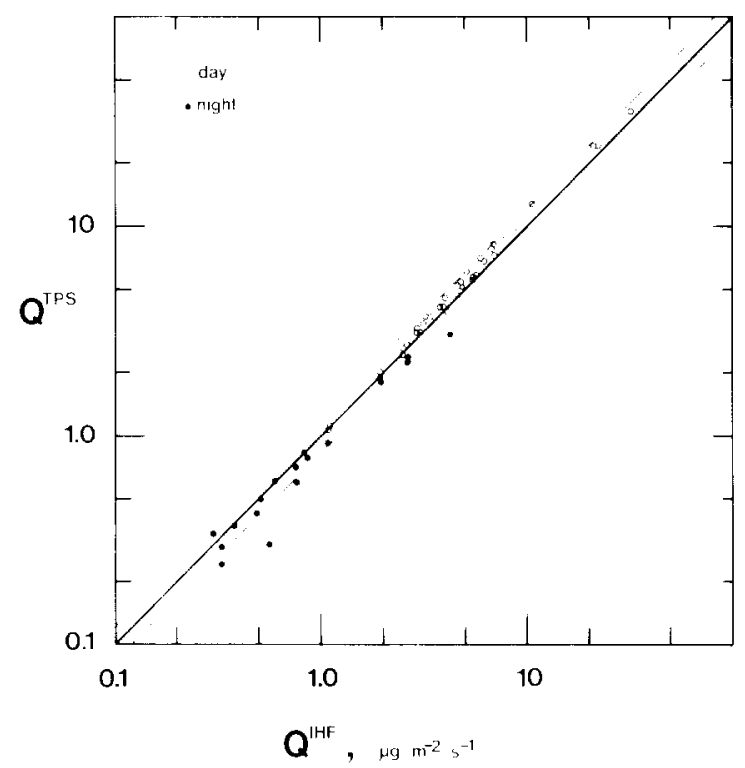

Fig. 4. A plot of $Q^{\mathrm{TPS}}$ versus $Q^{\mathrm{IHF}}$ for all 55 experiments. The dashed lines correspond to $Q^{\text {TPS }} / Q^{\mathrm{IHF}}=1.2,0.8$.

windspeed near the ground is small, the concentration is large, and the horizontal flux may not be negligible. Similarly above $z=2.5 \mathrm{~m}$ the concentration may be small, but the large windspeed implies a possibly significant horizontal flux. Graphical integration of the theoretical profiles shown in Fig. 2 (plotted in more detail to reveal the shape below $z=0.3 \mathrm{~m}$ ) yielded the result that the horizontal transport between $z=0.3 \mathrm{~m}$ and 
$z=2.5 \mathrm{~m}$ accounts for only $(76 \%, 81 \%, 84 \%)$ of the total emitted flux for the respective cases $L=(-5 \mathrm{~m}, \infty .+5 \mathrm{~m})$. Therefore, the above correction factors were applied to the source strength obtained by integrating the observed profile from $z=0.3 \mathrm{~m}$ to $z=2.5 \mathrm{~m}$, and the resulting value of the source strength was called $Q^{\mathrm{IHF}}$.

Figure 3 shows the time series of $Q^{\mathrm{IHF}}$ and $Q^{\mathrm{TPS}}$. The agreement is good, although it can be seen that $Q^{\text {TPS }}$ tends to be too high during the day and too low at night. The same behaviour is evident in Fig. 4, a plot of $Q^{\text {TPS }}$ against $Q^{1 \mathrm{HF}}$ for all 55 runs. However, as indicated by the dashed lines, only 5 of the 55 points correspond to a ratio $Q^{\mathrm{TPS}} / Q^{\mathrm{IHF}}$ differing from unity by more than 20\%. A least squares fit of $Q^{\text {TPS }}$ on $Q^{\mathrm{IHF}}$ gave $Q^{\mathrm{TPS}}=$ $1.13 Q^{\mathrm{IHF}}-0.216$ with a correlation coefficient of 0.998 . When the total $\mathrm{NH}_{3}$ loss over the $110 \mathrm{~h}$ is added up, the TPS estimate exceeds the IHF estimate by $8 \%$. This figure is biased by the high daytime value when $Q^{\text {TPS }}$ is too large. The average of the 552 -h values of $Q^{\mathrm{TPS}} / Q^{\mathrm{IHF}}$ was 1.01 (compensation of individual over-and under-estimates).

\section{CONCLUSION}

Neither the IHF nor the TPS estimate of ammonia volatilization is absolute, so that the above section gives a comparison of two estimates. Both methods ignore the contribution of the turbulent horizontal flux to the total horizontal flux at the centre of the plot (a contribution whose importance diminishes with increasing $R$ and which is probably $<10 \%$ of the total flux for these experiments). Further, it was not possible to perform the integration for $Q^{\mathrm{IHF}}$ without extrapolation above and below the range covered by the instruments. Nevertheless, $Q^{\mathbb{H F}}$ is probably a good estimate of the source strength.

The TPS method requires measurements of $\vec{s}$ and $\bar{c}$ only at a single height, but involves the additional (reasonable) assumption that the TS model is applicable (see WTKB for more detailed discussion). The TPS method has been found to agree with the IHF method as to the total loss of $\mathrm{NH}_{3}$ from the experimental plot over a 9 day period to within $8 \%$. Given the above-mentioned assumptions in application of the IHF method this discrepancy is not large. Therefore, the TPS method may be regarded as a satisfactory and easily implemented technique for determining the rate of loss from the ground to the atmosphere.

\section{REFERENCES}

Taylor, G.I., 1921. Diffusion by continuous movements. Proc. London Math. Soc. Ser. $2,20: 196-212$.

Wilson, J.D., Thurtell, G.W. and Kidd, G.E., 1981. Numerical simulation of particle trajectories in inhomogeneous turbulence, III : comparison of predictions with experimental data for the atmospheric surface layer. Boundary Layer Meteorol., 21: 443-463.

Wilson, J.D., Thurtell, G.W., Kidd, G.E. and Beauchamp, E.G., 1982. Estimation of the rate of gaseous mass transfer from a surface source plot to the atmosphere. Atmos. Environ., 16: 1861-1867. 